\title{
Design of Solar Power Management Circuit Based on Wireless Sensor Network
}

\author{
https://doi.org/10.3991/ijoe.v13i12.7891 \\ Jianying Shi $\left.{ }^{\varpi}\right)$ \\ Hebei University, Baoding, China \\ Yanbin $\mathrm{Xu}$ \\ Hebei College of Science and Technology, Baoding, China \\ ybxu209@126.com
}

\begin{abstract}
To explore the design of solar power management circuit, the fuzzy logic control algorithm based on MPPT, which has fast control speed and good environment robustness, is adopted as the control algorithm. In addition, the MPPT solar battery charge and discharge power management circuit is designed and successfully applied in the on-line measurement projects of dielectric loss of wireless sensor network in Jilin Province LG Electronics Company. The results show that the charging efficiency of solar battery charge and discharge power management circuit can reach above $80 \%$, and the current of static power management circuit is less than $1 \mathrm{~mA}$. In different light intensities, the dynamic power management is intelligently carried out. At last, it is concluded that the stability and reliability of circuit are quite high.
\end{abstract}

Keywords-Wireless sensor, power management, circuit design

\section{Introduction}

In the research on solar power supply, the control algorithms of MPPT in the past are divided into the following three categories: indirect control method based on parameter selection, direct control method based on sampled data, and intelligent control method based on modern control theory. Among them, the algorithm based on parameter selection is comparatively simple, but it cannot adapt to the influence of environment change on the parameters of solar panel. The control method based on sampled data is dynamic, which makes the maximum power point tracking according to environmental conditions, but the tracking is too slow. The MPPT algorithm based on the modern control theory makes up for the shortcomings of the above two algorithms, and it can achieve better maximum power point tracking. Compared with the previous two algorithms, this algorithm is more complex. Fuzzy logic control algorithm has the characteristics of fast control speed, good environment robustness and simple algorithm realization. As a result, it is chosen as the control algorithm for further study. 


\section{$2 \quad$ Literature review}

Because the wireless sensor network technology has been developed earlier abroad, there are more related solar power supply circuit systems. For example, Prometheus has designed a power supply circuit based on solar photovoltaic panels charging super capacitors and lithium batteries in two stages' storage. The first stage uses super capacitors to store energy, and the second stage uses lithium batteries to store energy. The management circuit in the circuit is mainly responsible for managing the charging and discharging of the lithium batteries, but cannot carry on the MPPT to the solar energy, and has not realized the full use of energy [1]. Compared with Prometheus system, C. Park and some scholars designed AmbiMax, which makes use of the method of maximum power point tracking to store the solar panel output energy in the capacitor, and the system also collects wind power for the charging of the super capacitors [2,3]. However, due to the large capacity of the selected super capacitors, the leakage phenomenon of the super capacitors is also very serious, and the system does not deal with the leakage of super capacitors very well. In view of the leakage problem of super capacitors, Twinstar system adopts leakage-induced feedback control technology and energy synchronization technology, and basically realizes the long-lasting power supply of super capacitor power supply node [4]. For the power management circuit of wireless sensor nodes studied, its main purpose is to implement the following three aspects of functionality: to achieve the maximum efficiency of the system's input energy and the solar panels' output energy; to achieve the most efficient and the most reasonable allocation of system's output to the system's load and battery; and to minimize the power management circuit energy consumption.

\section{Power management circuit software design}

\subsection{MPPT control algorithm design}

MPPT control algorithm, namely the maximum power point tracking algorithm, is sampled by the output current of DCDC conversion circuit. By adjusting the duty ratio of PWM output, the accurate tracking of the maximum power point of solar panels can be realized. It will be the best to achieve the maximum power point tracking by using efficient algorithm. The more streamlined the algorithm is, the faster the implementation of the maximum power point tracking is, and the smaller the corresponding power consumption is.

According to the requirements proposed in this paper, the control parameters are the output current of DCDC circuit. Based on this, the fuzzy linguistic variables of the corresponding bias can be set to $\mathrm{x}$, and the fuzzy domain is [-50 50]. The fuzzy linguistic variables of error rate are $y$, and the fuzzy domain is [-100 100]; the output linguistic variables are $\mathrm{dD}$, and the fuzzy domain is [-50 50]. The unit corresponding to the fuzzy domain is mA. Here we define three fuzzy variables: the deviation X, the deviation change rate $\mathrm{y}$, and the output control quantity $\mathrm{dD}$ [5]. They are made up of 8 fuzzy language values, $\{\mathrm{NB}, \mathrm{NM}, \mathrm{NS}, \mathrm{N} 0, \mathrm{P} 0, \mathrm{PS}, \mathrm{PM}, \mathrm{PB}\}$, which correspond to 
\{negative big, negative middle, negative small, negative 0 , positive 0 , positive small, positive middle, and positive big\} respectively. According to the definition of fuzzy variables mentioned above, the fuzzy logic rule table corresponding to the maximum power point tracking algorithm adopted in this paper is shown in Table 1.

Table 1. Maximum Power Point Tracking Fuzzy Control Rule

\begin{tabular}{|c|c|c|c|c|c|c|c|c|c|}
\hline \multicolumn{2}{|c|}{$\begin{array}{c}\text { The maximum } \\
\text { value }\end{array}$} & \multicolumn{9}{|c|}{$\mathbf{X}$} \\
\cline { 2 - 10 } & $\boldsymbol{N B}$ & $\boldsymbol{N M}$ & $\boldsymbol{N S}$ & $\boldsymbol{N O}$ & $\boldsymbol{P O}$ & $\boldsymbol{P S}$ & $\boldsymbol{P M}$ & $\boldsymbol{P B}$ \\
\hline \multirow{6}{*}{} & NB & PB & PM & PS & PO & NO & NS & NS & NB \\
\cline { 2 - 10 } & NM & PR & PM & PS & PO & NO & NO & NS & NS \\
\cline { 2 - 10 } & NS & PM & PS & PS & PO & NO & NO & NO & NS \\
\cline { 2 - 10 } & NO & PS & PS & PO & PO & NO & NO & NO & NO \\
\cline { 2 - 10 } & PO & NO & NO & NO & NO & NO & NO & NO & NO \\
\cline { 2 - 10 } & PS & NS & NO & NO & NO & PO & PS & PS & PM \\
\cline { 2 - 10 } & PM & NS & NS & NO & NO & PO & PS & PM & PB \\
\cline { 2 - 10 } & PR & NB & NS & NS & NO & PO & PS & PM & PB \\
\hline
\end{tabular}

\subsection{Design of charge control algorithm for lead acid battery}

For the constant current and constant voltage charging battery algorithm, the dualmode control algorithm with parallel connection of fuzzy control [6] and PI control is adopted. It can not only make the fuzzy control algorithm well adapt to the change of the external environment, but also can eliminate the residual error existing in the fuzzy logic control algorithm and thus realize the optimized control of battery charging with constant current and constant voltage. Fuzzy-PI dual-mode control algorithm is a parallel dual-mode control algorithm and its control principle is that when the input error is great, the fuzzy control algorithm with fast adjustment speed is used to control and get better performance in dynamic adjustment [7]. When the input error is small, the PI integral control is adopted, and through eliminating the residual error of the control system as a whole, better steady-state performance can be obtained. The algorithm diagram is shown in Figure 1.

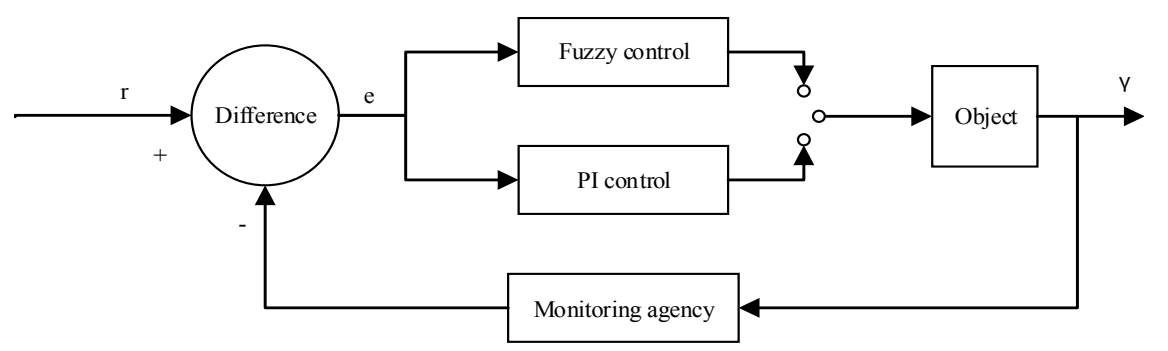

Fig. 1. Fuzzy-PI Dual Mode Control System Structure

When e is greater than E, the fuzzy control is carried out; when e is less than E, the PI is controlled ( $E$ is the set error threshold). 
The design of fuzzy controller charging with constant voltage and constant current: for the fuzzy controllers with constant current and constant voltage, the control parameters are output current or output voltage of the DCDC circuit. Then, the fuzzy linguistic variable of corresponding deviation can be set to e, the corresponding domain is [-10 10]. The fuzzy linguistic variable of error change rate is ee, and the corresponding domain is [-30 30]. The output language variable is $\mathrm{dD}$, and the corresponding domain is $[-5050]$. The unit corresponding to the fuzzy field is $\mathrm{mA}$ or $\mathrm{mV}$. Here we define the deviation e and deviation change rate ee. The output control $\mathrm{dD}$ is composed of 7 fuzzy linguistic values, $\{\mathrm{NB}, \mathrm{NM}, \mathrm{NS}, \mathrm{ZE}, \mathrm{PS}, \mathrm{PM}, \mathrm{PB}\}$, which correspond to \{negative big, negative middle, negative small, zero, positive small, positive middle, positive big\} respectively [8]. According to the above definition of fuzzy variables, fuzzy control algorithm rules on charging with constant voltage and constant current are shown in Table 2.

Table 2. Fuzzy Control Algorithm Rules on Charging with Constant Voltage and Constant Current

\begin{tabular}{|c|c|c|c|c|c|c|c|c|}
\hline \multicolumn{2}{|c|}{ The maximum value } & \multicolumn{7}{|c|}{ e } \\
\cline { 2 - 9 } \multicolumn{1}{|c|}{} & $\boldsymbol{N B}$ & $\boldsymbol{N} \boldsymbol{M}$ & $\boldsymbol{N} \boldsymbol{S}$ & $\boldsymbol{Z} \boldsymbol{E}$ & $\boldsymbol{P S}$ & $\boldsymbol{P} \boldsymbol{M}$ & $\boldsymbol{P} \boldsymbol{B}$ \\
\hline \multirow{4}{*}{ ee } & NB & PB & PB & PB & PB & PS & PS & ZE \\
\cline { 2 - 9 } & NM & PB & PB & PB & PM & PS & ZE & NS \\
\cline { 2 - 9 } & NS & PB & PB & PM & PS & ZE & NS & NM \\
\cline { 2 - 9 } & ZE & PB & PM & PS & ZE & NS & NM & NB \\
\cline { 2 - 9 } & PS & PM & PS & ZE & NS & NM & NB & NB \\
\cline { 2 - 9 } & PM & PS & ZE & NS & NM & NB & NB & NB \\
\cline { 2 - 9 } & PB & ZE & NS & NM & NB & NB & NB & NB \\
\hline
\end{tabular}

\subsection{Power management control software design}

The power management control software mainly includes the running and sleeping state of the circuit, the switching of the battery charging mode, and the discharge control of the battery. In order to reduce the energy consumption of the power management circuit, the power management circuit itself has two operating states: running state and sleeping state.

The running state of power: when the solar panel's output energy is higher than the power of the power management circuit in the running state, the power management circuit operates on the MPPT tracking of the solar panel's output energy, with charging for lead-acid battery under constant current or constant voltage. It also maintains the lead-acid battery in floating state or maintains solar panels in direct power supply state.

The sleeping state: when the output power of the management circuit is less than the consumption of the power management circuit in the running state, the power management circuit enters a dormant state, so as to reduce the energy consumption of the power management circuit itself to the storage battery. After the power management circuit enters the dormant state and reaches a certain value at the output voltage 
of the solar panel, it will trigger the external interruption of microcontroller in the power management circuit, so the power management circuit began to wake up and work. The basic flow of the power management control software is shown in Figure 2 .

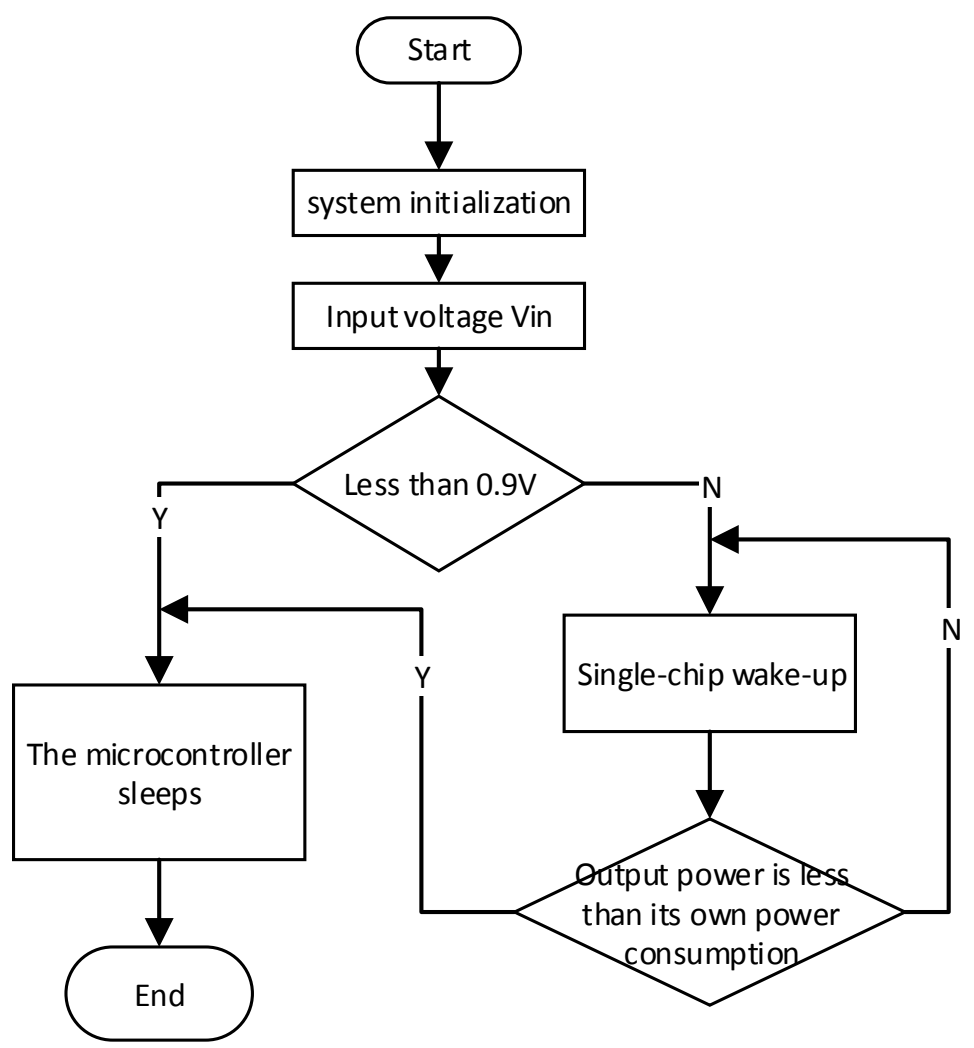

Fig. 2. Flow Chart of Power Supply Control Software

As the power management system studied in this paper has several tasks, such as AD sampling, MPPT, constant current charging, constant voltage charging, discharge control, serial communication, and watchdog monitoring. In order to improve the real-time nature, readability and expansibility of management software, this paper chooses to transplant the power management software to real-time operating system based on STM8 design for the scheduling management of multiple tasks, and uses STM8 officially configured RTOS system atom threads. Atom threads is a free, lightweight, portable, and real-time scheduling embedded operating system. It uses BSD licenses to publish source code, and can be used for free, whether for commercial or educational purposes. Atom threads addresses a system that requires only schedulers and basic RTOS functionality, and it does not include file systems, IP stacks, or device drivers. But developers can add as necessary. Atom threads is a small $\mathrm{C}$ file that is easily ported to other platforms. 


\section{Experimental data and results of power management circuits}

\subsection{MPPT experimental data}

In the MPPT experiment, the solar power management circuit is connected with $4.5 \mathrm{~V}$ and $3 \mathrm{~W}$ solar panel input DCDC converter, for measuring the output power Pin of photovoltaic cells and converter output current lout in different proportion factors $\mathrm{k}$, and the curve obtained from the experiment is shown in Figure 3. In the experiment, the environmental parameters are light intensity of 45700LUX and temperature of 5 DEG C. According to the curve in Figure 3, it is known that, with the increase of $\mathrm{k}$ value, the output power of photovoltaic cell and the output current of converter are both increased first and then decreased, and the maximum value is reached under the same condition of $\mathrm{k}=0.8$ [9]. The experimental data show that the tracking of the maximum output power point (the maximum power point) of the solar panel can be realized by detecting the output current of the converter and by adjusting the $\mathrm{k}$ value.

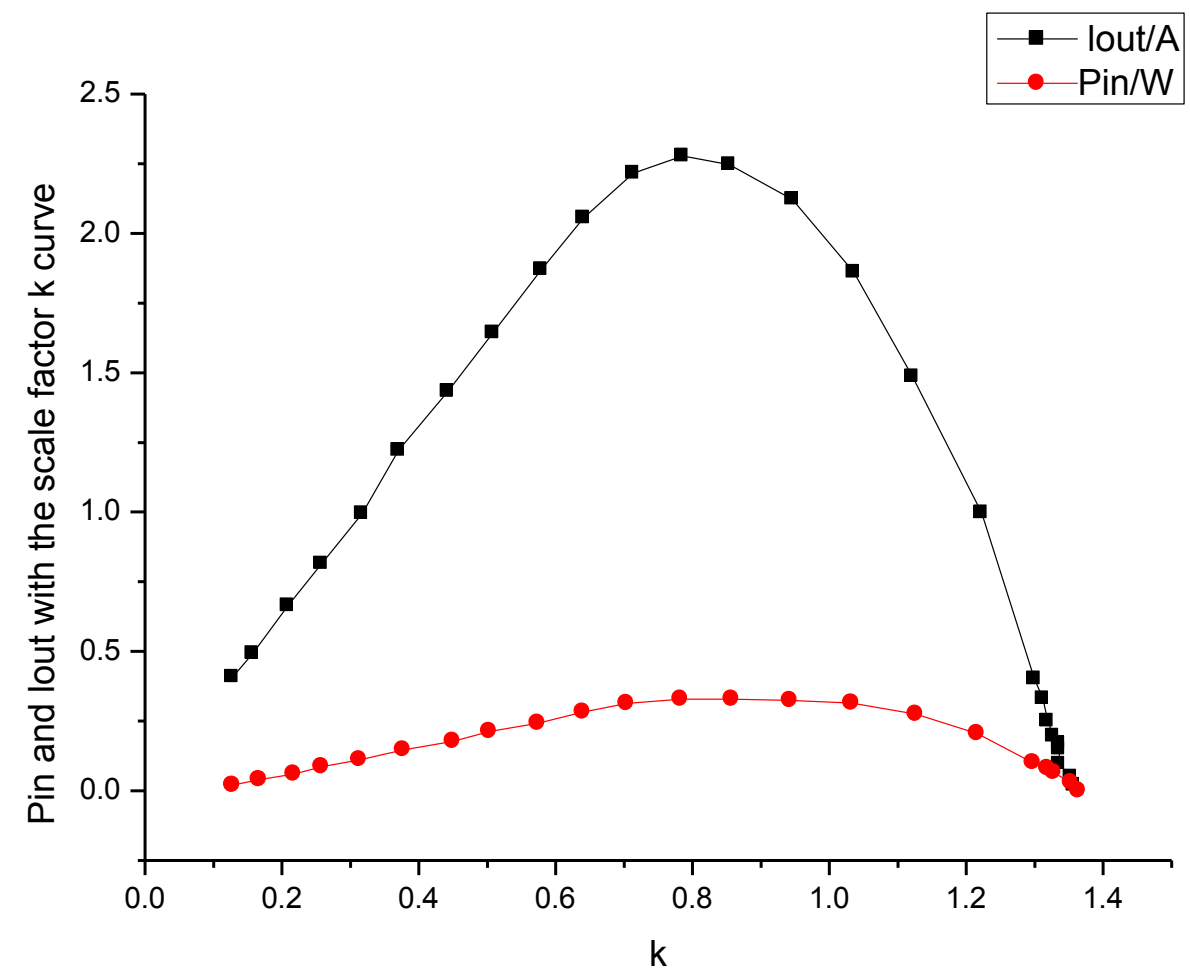

Fig. 3. Curve of Solar Panel Input Power Pin and DCDC Circuit Output Current Lout Changing with $\mathrm{k}$ 


\subsection{Charging experimental data}

In this paper, a comparative experiment is made on the MPPT charging effects of power management circuit. The experiment uses the same solar panel to charge a lead-acid battery. The charging circuit selects a power management circuit studied in this paper and an integrated solar charging management chip CN3082. The charging chip does not have the function of MPPT. The two, under the same environmental conditions, through the same battery panel, compare the charging current on the same battery, and the experimental data is shown in Figure 4.

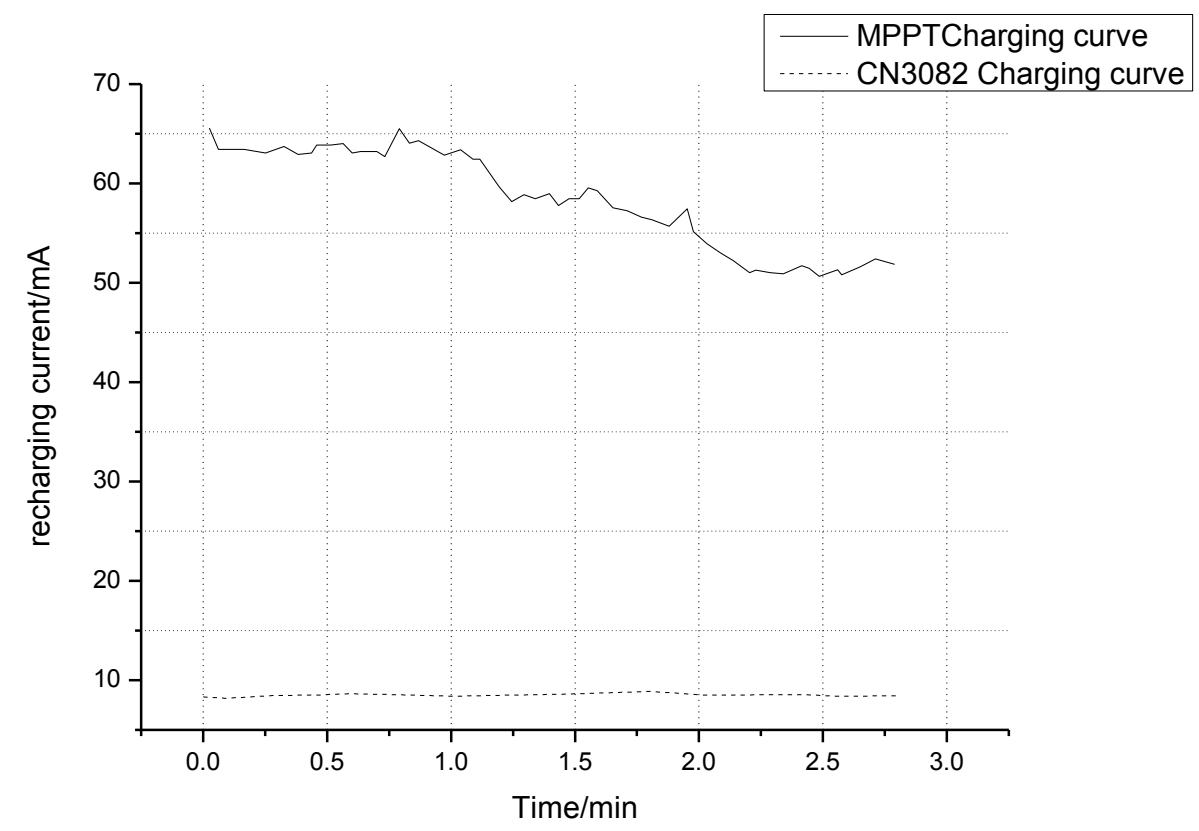

Fig. 4. Contrast Experimental Data between MPPT Charging and CN3082 (nonMPPT) Charging

Figure 4 is a comparative experiment of the charging current of lead-acid batteries at about 9 a.m. on May 31, 2017 when the ambient temperature was under the light intensity of 28200LUX, and the temperature was 25V [10-12]. From the curve in Figure, it can be seen that, under the premise of the same environment, the charging current of MPPT tracking charging circuit can reach about 3 times of the charging current of CN3082. It indicates that the charging mode of MPPT tracking can better make full use of the output energy of the solar battery panel, as well as use of solar energy. 


\section{Conclusions}

This paper designs a charging and discharging power management circuit of MPPT solar power battery, and the charging efficiency can reach above $80 \%$. The static working circuit of power management circuit is less than $1 \mathrm{~mA}$, and it can make intelligent dynamic power management in different light intensities, and both stability and reliability of circuit are quite high.

It only focuses on power management circuit of low power (within $5 \mathrm{~W}$ ) solar power panel. If there is an opportunity, the design of power management circuit of high power (10W-500W) solar power panel would be explored. The control algorithm studied here can be easily transplanted into the power management circuit for highpower solar panels. And the solar power system with high power can be applied in the camera collection in some field work, $4 \mathrm{G}$ wireless transmission and other projects.

\section{$6 \quad$ References}

[1] Climent, S., Sánchez, A., Blanc, S., Capella, J. V., \& Ors, R. (2016). Wireless sensor network with energy harvesting: modeling and simulation based on a practical architecture using real radiation levels. Concurrency and Computation: Practice and Experience, 28(6): 1812-1830. https://doi.org/10.1002/cpe.3151

[2] Yoon, I., Kim, H., \& Noh, D. K. (2017). Adaptive Data Aggregation and Compression to Improve Energy Utilization in Solar-Powered Wireless Sensor Networks. Sensors, 17(6): 1226. https://doi.org/10.3390/s17061226

[3] Chowdary, G., Singh, A., \& Chatterjee, S. (2016). An 18 nA, 87\% Efficient Solar, Vibration and RF Energy-Harvesting Power Management System With a Single Shared Inductor. IEEE Journal of Solid-State Circuits, 51(10): 2501-2513. https://doi.org/10.1109/ JSSC.2016.2585304

[4] El-Damak, D., \& Chandrakasan, A. P. (2016). A $10 \mathrm{nW}-1 \mu \mathrm{W}$ Power Management IC With Integrated Battery Management and Self-Startup for Energy Harvesting Applications. IEEE Journal of Solid-State Circuits, 51(4): 943-954. https://doi.org/10.1109/JSS C.2015.2503350

[5] Hosseinzadeh, M., \& Salmasi, F. R. (2016). Determination of maximum solar power under shading and converter faults - a prerequisite for failure-tolerant power management systems. Simulation Modelling Practice and Theory, 62: 14-30. https://doi.org/10.1016/j.sim pat.2016.01.011

[6] Bayrak, Z. U., Bayrak, G., Ozdemir, M. T., Gencoglu, M. T., \& Cebeci, M. (2016). A lowcost power management system design for residential hydrogen \& solar energy based power plants. International Journal of Hydrogen Energy, 41(29): 12569-12581. https://doi.org/10.1016/j.ijhydene.2016.01.093

[7] Chen, N., Jung, H. J., Jabbar, H., Sung, T. H., \& Wei, T. (2017). A piezoelectric impactinduced vibration cantilever energy harvester from speed bump with a low-power power management circuit. Sensors and Actuators A: Physical, 254: 134-144. https://doi.org/10.1016/j.sna.2016.12.006

[8] Aktas, A., Erhan, K., Ozdemir, S., \& Ozdemir, E. (2017). Experimental investigation of a new smart energy management algorithm for a hybrid energy storage system in smart grid applications. Electric Power Systems Research, 144: 185-196. https://doi.org/10.1016/ j.epsr.2016.11.022 
[9] Zessin, H., Spies, P., \& Mateu, L. (2016, November). Power density improvement of the power conditioning circuit for combined piezoelectric and electrodynamic generators. In Journal of Physics: Conference Series (Vol. 773, No. 1, p. 012055). IOP Publishing. https://doi.org/10.1088/1742-6596/773/1/012055

[10] Pukhrem, S., Basu, M., Conlon, M. F., \& Sunderland, K. (2017). Enhanced Network Voltage Management Techniques Under the Proliferation of Rooftop Solar PV Installation in Low-Voltage Distribution Network. IEEE Journal of Emerging and Selected Topics in Power Electronics, 5(2): 681-694. https://doi.org/10.1109/jestpe.2016.2614986.

[11] Guo, K. (2016). Empirical Study on Factors of Student Satisfaction in Higher Education. Revista Iberica de Sistemas e Tecnologias de Informacao, E11: 344-355.

[12] Duan, H.Y. (2016). Research on Collaboration in Innovative Methods of Manufacturing Innovation Chain. Revista Iberica de Sistemas e Tecnologias de Informacao, E11: 292303.

\section{Authors}

Jianying Shi is an Associate Professor at Electronic Information Engineering College, Hebei University, Baoding 071002, China (e-mail: hdsjy@126.com).

Yanbin Xu is an Associate Professor at Department of Electrical Engineering, Hebei College of Science and Technology, Baoding 071000, China (e-mail: ybxu209@126.com).

Article submitted 25 October 2017. Published as resubmitted by the authors 30 November 2017. 